\title{
LA FLOR DE LA JACARANDINA
}

\author{
Con el recuerdo presente \\ de las jacarandas de San Ángel
}

La expresión "flor de la jacarandina" - y/o su variante "flor de la jacarandaina" - aparece documentada en dos conocidísimas obras de mediados del siglo xvir, El alcalde de Zalamea y la Vida y hechos de Estebanillo González, escritas al parecer a muy pocos años de distancia. Si nos atenemos exclusivamente a las fechas de publicación, no cabe duda de que la prioridad le corresponde al $E s$ tebanillo. Éste se publica, según es sabido, en 1646, mientras que la princeps del Alcalde es de 1651. Ahora bien, según también es harto conocido y según ocurre a cada paso en el terreno de la producción teatral, ésta no es más que una fecha adquem, y prevalece la opinión de que la redacción de la obra tuvo que ser mucho más temprana ${ }^{1}$. Lo que da interés a la averiguación de si el drama calderoniano se llegó a representar antes de 1646 es la aparición, en el capítulo III de la narración de Estebanillo, de otra coincidencia textual con el interludio musical intercalado en la primera jornada de El alcalde (vv. 101-112), interludio en el que cantan alternativamente la Chispa y Rebolledo, cada cual con tres intervenciones paralelas de dos versos ${ }^{2}$. Con una variante que afecta

1 Véase, a este propósito, la introducción de José María Díez Borque a su edición, Clásicos Castalia, Madrid, 1987, pp. 55-57.

${ }^{2}$ Lo que de momento me interesa resaltar es la simetría de sus intervenciones, aunque, según veremos, también es altamente significativo el cambio métrico que hace pasar de los dos pareados del principio al romance en cuartetas que sigue. Puede observarse que el sintagma "flor de la jacarandaina", que Estebanillo se aplica a sí mismo en los primeros renglones de su prólogo, figura en el pareado con el que la Chispa da comienzo al conjunto de las intervenciones líricas de $E l$ alcalde, mientras que los versos de romance que, más adelante, tienen la particularidad de coincidir nuevamente con otro fragmen- 
exclusivamente al sexo de la persona interpelada, se cantan en efec to en este dúo y se citan en el ya referido capítulo III del Estebani llo los siguientes versos: "Huéspeda [Huéspede], máteme una ga. llina/que el carnero me hace mal'". Frente a la existencia de esta: dos coincidencias textuales, son varias las hipótesis que se pue den formular. Cabe, en primer lugar, la posibilidad de que nc sean significativas y de que sólo se deban a un curioso azar. Di no considerarse esto plausible, puede pensarse que el autor anó nimo del Estebanillo tuvo presente el dúo de la Chispa con Rebo lledo. Nada, sin embargo, autoriza a descartar la idea de que fuer: Calderón quien, al revés, tomase como punto de partida para li elaboración de su propio interludio musical una autobiografía cuy: primera edición española llevaría, en 1652, una autorización fu mada de su mano ${ }^{3}$. Cabe pensar, por último, que ambos auto res partan de una fuente común, hoy desconocida. La consult: del valioso índice puesto ahora a la disposición de todos por An tonio Carreira y Jesús Antonio Cid permite observar que son abun dantísimos los casos en que los dos autores en cuestión están acu diendo al mismo fondo común de dichos festivos y reminiscencia romanceriles o proverbiales. Pero, gracias a la meticulosa anota ción de los mismos editores, también se observa con facilidad que si se exceptúan las dos coincidencias que aquí me interesan, la demás no autorizan a hablar de intertextualidad, puesto que este equivaldría a aplicar este concepto al uso de bienes mostrencos aprovechados igualmente por dos o tres generaciones de escri tores ${ }^{4}$.

No se ha tomado hasta ahora aquí en cuenta otro texto, mu cho menos conocido que los anteriores, en el que también figur el sintagma nominal que ha servido de punto de partida para lai presentes reflexiones. Como para que no quedara ninguna variant posible fuera del cortísimo catálogo que de dicho sintagma se pued

to del mismo interludio ("huéspede, máteme una gallina,/que el carnero me hace mal", La vida y los hechos de Estebanillo González, eds. Antonio Carreirc y Jesús Antonio Cid, Cátedra, Madrid, 1990, p. 146) corresponden, por e contrario, al cierre del dúo calderoniano.

${ }^{3}$ Véase la ed. cit., pp. xxii y 5.

${ }^{4}$ Remito en particular a los ejemplos aducidos en I, 138, n. 81; II, 9 n. 10; II, 44, n. 152; II, 188, n. 134. Carreira y Cid señalan, por otra parte que entre Calderón y el autor de la Vida de Estebanillo existen, al margen dt otras coincidencias, un número importante de convergencias en el terreno dt la "ideología" (son ellos quienes hacen uso de las comillas). Véase su intro ducción, p. xxii, n. 3. 
ofrecer, lo que se documenta es en este caso lo siguiente: "Nana, nina, dina, dana/flor de la jacarandana./Dina, dana, dana, dina,/flor de la jacarandina". La canción que se acaba de citar figura en un baile que permaneció inédito hasta una fecha relativamente reciente, aunque Cotarelo da noticia de su existencia y ofrece un resumen de su contenido ${ }^{5}$. El dato se recoge, como era de esperar, en el monumental Corpus de Margit Frenk, donde se reproduce el texto completo de la canción ${ }^{6}$. Ahora bien, este baile ha sido editado en 1986 por María Luisa Lobato, quien lo presenta con muy sólidos argumentos como la segunda parte de Las jácaras, entremés atribuido a Calderón, y defiende la idea de que ambas piezas se representaron por primera vez hacia $1640^{7}$. Es cierto que la novedad de estos datos no permite de ningún modo averiguar hasta qué punto estaba, o no, lexicalizado el sintagma flor de la jacarandina, con sus ya mencionadas variantes, ni si la tal lexicahzación - en el caso de que aceptemos darla por ciertaes fenómeno propio de los años $1640^{8}$. Como a continuación procuraré mostrar, estos datos son en cambio de mucho interés para quien se propone averiguar qué es lo que exactamente significa su empleo cuando se le asigna el decorativo papel que desempeña al comienzo del drama calderoniano.

${ }^{5}$ Emilio Cotarelo y Mori, Colección de entremeses, loas, bailes, jácaras y mogigangas desde fines del siglo xvi a mediados del xviii, $N B A E$, Madrid, 1911, t. 17, p. cccii.

${ }^{6}$ Margit Frenk, Corpus de la lírica antigua popular hispánica (siglos xv a xvii), Castalia, Madrid, 1987, núm. 1560.

7 "Segunda parte inédita del entremés Las jácaras atribuido a Calderón", RILCE, 1 (1986), 119-140. Véanse ahora el texto y los argumentos a favor de la doble atribución en: CALDERón, Teatro cómico breve, Reichenberger, Kassel, 1989 , pp. 369-374 y 392-409. Para la fecha probable de la primera representación, véase la p. 370 .

${ }^{8}$ Adviértase que en uno de los romances de la famosa recopilación de Juan Hidalgo figura ya una alusión a las "flores que hay en la jacarandina" (“ ‘Ay coima, la más godeña/de toda la germanía,/por quien mi brazo derecho/ha mostrado su valía [...]/usando por ti más flores/que hay en la jacarandina!", en JoHN M. HiLl, Poesías germanescas..., Indiana University Press, Bloomington, 1945, p. 76, núm. XXX). Aunque el característico uso germanesco de flores, en plural, sólo está muy remotamente en relación con el de la misma palabra, en singular, para designar hiperbólicamente la crema de la germanía, esta asociación temprana de las dos palabras claves que figuran en el sintagma flor de la jacarandina puede interpretarse como indicio de una lexicalización precoz del mismo, al que se remitiría festivamente en el romance que acabo de citar; pero también puede pensarse que este divulgadísimo romance contribuyó, por el contrario, a que se inventara la expresión hiperbólica que aquí me interesa. 
Lo primero que quiero resaltar es que, si se admite la atribución propuesta por María Luisa Lobato, son dos los casos en que una canción, en la que unas muy llamativas variaciones onomatopéyicas alternan juguetonamente con referencias asonantadas a la flor de la jacarandanaljacarandainaljacarandina, se usa en Calderón a modo de introducción casi arquetípica al mundo de la jácara. Vemos en efecto que acuden espontáneamente a ella, cuando se ven incitadas a dar una muestra de su maestría en este terreno, tanto la hija caprichosa que, en la Segunda parte de Las jácaras, se niega a satisfacer a su padre volviendo a jacarear a cada paso, según se indica que hasta entonces solía hacer, como la Chispa que, en El alcalde, goza de la misma fama. Nos encontramos, pues, ante una perfecta ilustración de la situación descrita por José Luis Alonso cuando, al discutir el papel tradicionalmente asignado a Quevedo en la evolución de la jácara, afirma que, lejos de ser el que "la hace nacer", es quien la entierra, explotando las posibilidades del género de una manera exacerbada, conforme a su costumbre, y contribuyendo así de manera decisiva a que éste quede, en lo sucesivo, "vaciado del lenguaje de los que le dan nombre", "desdramatizado" e "invadido por el baile" con "un aumento del aspecto musical en detrimento de los demás"9. Prosiguiendo su análisis, observa José Luis Alonso que, mientras va desapareciendo la jácara-germana, que se distinguía en particular por lo críptico de su lenguaje, la tonadilla y el baile, a los que su metamorfosis deja el camino abierto, se van como a modo de compensación llenando de "términos con regusto americano o exótico en general destinados sobre todo a puntear el ritmo", 10 .

La solución por la cual opta Calderón, tanto en la Segunda parte de Las jácaras —en el caso en que sea realmente suya-, como en el interludio cantado a dos voces por Rebolledo y la Chispa no es la del exotismo, sino la de unos juegos fonéticos de inconfundible sabor tradicional, cuyo vocalismo es llamativamente afín al del guilindín, guilindín, guilindaña seleccionado por Margit

${ }^{9}$ José Luis Alonso Hernández, "Los lenguajes de la jácara en su metamorfosis", en El teatro español a fines del siglo xvii. Historia, cultura y teatro en la España de Carlos II. (Diálogos Hispánicos de Amsterdam, 8/II), Rodopi, Amsterdam, 1989, p. 620. Sobre la progresiva reducción de la materia hampesca (impropiamente llamada "picaresca") y la invasión de la jácara por la música y el baile, véase también lo dicho, en el mismo volumen, por Eva SÁNCHEz FERnÁndez-Bernal, "Algunas notas sobre la jácara dramática", p. 594.

${ }^{10}$ Las muestras aducidas en nota para ilustrar estas palabras son las que siguen: jíquiri-juaico, charupa, chamaria, pitibú, turumbi, chiqui, rufa y fa, lanturlú, lantaralú, paracumbé. 
Frenk para encabezar el apartado de su Corpus en el que estos juegos se recogen en su gran mayoría ${ }^{11}$. Pese a lo cual, creo que los términos del análisis de José Luis Alonso siguen siendo válidos para medir, en el caso de los versos 101-104 de El alcalde ("Yo soy, tiri, tiri, taina,/flor de la jacarandaina./Yo soy, tiri, tiri, tina,/flor de la jacarandina'"12), lo tenue de su relación con la tradición germana. O, para decirlo con palabras tal vez más adecuadas, para valorar el alto grado de estilización con el que esta tradición está allí elípticamente aprovechada. Si se examina el conjunto de los versos cantados por la Chispa y por su compañero, resulta en efecto fácil advertir que el aparatoso empleo de los sintagmas flor de la jacarandainalflor de la jacarandina, que alternan en los pareados del principio, es lo único que autoriza a hablar a su propósito de clave hampesca. El resto de la canción no se sitúa ya ni lingüística ni temáticamente en esta clave. Quien tenga dudas, vuelva a leer las dos cuartetas en las que, con significativa reiteración de algunas palabras aplicadas, según el caso, a la vida militar o a la comida, se celebra el rechazo de toda empresa bélica, incluso "santiaguista" y se exalta, por contraste, la adhesión a un ideal de vida muy parecido al del proverbial rey $\mathrm{Pa}$ lomo: "Vaya a la guerra el alférez,/y embárquese el capitán./Mate moros quien quisiere,/que a mí no me han hecho mal./Vaya y venga la tabla al horno,/y a mí no me falte pan./Huéspeda, máteme una gallina,/que el carnero me hace mal".

Tal vez la aguda conciencia de que esta canción no es de ninguna manera una jácara sea lo que explique que, cuando la Chispa se ve instada a cantar para aliviar la fatiga de la tropa (vv. 9094), se le pide de un modo significativo que interprete "una jácara o canción". Creo que autorizan a interpretar así el detalle no sólo la consideración de los incontables casos en los que sabemos que Calderón está de un modo parecido llamando la atención sobre alguna particularidad de su quehacer poético sino, mucho más concretamente, y tratándose de jácaras, la del entremés y del baile que con este título figuran ahora en la recopilación de su teatro cómico breve. Aunque un estudio completo del tema requeriría

11 Véanse los núms. 1503 ss.

${ }^{12}$ Sigo aquí la versión de la princeps, reproducida por Díez Borque en su edición. La que se recoge en el Corpus de la antigua lirica popular (títiri, títiri, tina/titiri, taina) corresponde a la presentada por Vera Tassis. La opción es claramente entre la prioridad dada a una métrica regular (solución de la princeps, seguida por Díez Borque), o la aceptación de una irregularidad al parecer más acorde con el ritmo propio de la lírica de tipo popular. 
que el juego calderoniano con la poética de la jácara-germana se exa minara sucesivamente en estas dos piezas, puesto que se presentí en la una y en la otra con interesantes variantes, me limitaré ć recordar aquí cuáles son las bases sobre las que este juego se asient en el entremés. El personaje central de dicho entremés, Marizar. pa, tiene desesperado a su padre por su enloquecida afición a cantas jacaras, que ha llegado a interpretar, con acompañamiento de cas. tañetas, "noche y día", sin interrupción. El remedio, ideado por el gracioso, consistirá en hacer que los jaques o las izas cuyos nombres se sacan a relucir, conforme a las normas del género, en los primeros versos de las jácaras que Marizarpa se pone a cantar. se le aparezcan protestando y exigiendo, con amenazas, que les deje en adelante descansar en paz. Lo que en este caso se propone es, pues, otro tratamiento estilizado de la tradición germana de representación festiva de la vida hampesca, aunque conseguido con recursos polarmente opuestos a los que encontramos en la primera intervención cantada de la Chispa, en El alcalde. Lejos de rechazarse por completo la narratividad épica de signo rebajador y denigrante, que encontramos en los poemas que celebran las hazañas, pendencias, amores o muertes de marcas y rufianes, se crean condiciones para que sobre estos temas vayan acumulándose las proposiciones narrativas, pero con la particular circunstancia de que ninguna de ellas llega a alcanzar su pleno desarrollo. El resultado al que se llega es, al fin y al cabo, tan demoledor para la presentación de vidas o de fragmentos de vidas hampescas como resulta serlo la solución inversa de completa omisión del tratamiento antiépico supuestamente de rigor.

La alusión que acabo de hacer a las jácaras del entremés, de las cuales ninguna se llega a cantar hasta el final, posiblemente haya despertado ecos familiares en quienes recuerdan que la jácara de tipo germanesco, de la que no hay ejemplo en la primera jornada de El alcalde de Zalamea, se inserta en cambio muy logradamente en la segunda jornada. Con su interpretación, que tampoco llega a pasar de los quince primeros versos, culmina en efecto la serie de contrastes que se introducen en dicha jornada, a partir del momento en que la paz del jardín de Crespo, en la que sólo sonaba la música del agua en la fuente, se encuentra turbada por la agresiva imposición de otro tipo de música. Es obvio que la violencia de la situación, señalada desde el momento inicial de la serenata por los apartes de cuatro de los cinco personajes que ocupan entonces el escenario, alcanza un clímax intolerable con la canallesca intrusión de la jácara cantada por la Chispa. En la 
medida en que he llamado antes la atención sobre el curioso uso de dos palabras que se observa en la primera jornada, cuando se le pide a la Chispa que interprete "una jácara o canción', , completo ahora la información, advirtiendo que el uso de los dos verbos correspondientes, cantar y jacarear, vuelve a figurar en las proposiciones hechas por Rebolledo, en el momento en que éste prepara con el Capitán el programa de la música nocturna que se proponen interpretar debajo de los balcones de Isabel ${ }^{13}$. En este segundo caso, sin embargo, la aplicación respectiva de los dos vocablos responde programáticamente a una distinción semántica muy clara: a la poética canción que encierra una calculada referencia directa al nombre de la joven a quien va dirigida la serenata, y forma al mismo tiempo auténticamente parte del acervo de la lírica tradicional ("Las flores del romero,/niña Isabel,/hoy son flores azules,/y mañana serán miel"'14), sucede en efecto la presentación de un trío rufianesco que suena casi a pastiche de la jácara-germana de comienzos de siglo ("Érase cierto Sampayo,/la flor de los andaluces,/el jaque de mayor porte/y el rufo de mayor lustre", etc.) Queda clarísimo que la crudeza de la jácara anula por completo los efectos del halo poético que la canción tradicional conserva, pese a las tensiones en medio de las cuales se canta, dando así claramente a entender que la perspectiva de un dulce mañana hecho de miel, que promete la canción, no tendrá cabida en el drama en el que esta canción se inserta.

Puede pensarse que el hecho de que no se canten jácaras ni antes ni después de este momento crucial de la obra, pese a haberse dado desde el principio a entender que en este terreno es donde suele brillar la Chispa, se debe en cierta medida a razones de equilibrio. Para que la muestra del género que se nos presenta en medio del drama conservara su virulencia y su aspecto provocador, es obvio que era preciso que conservara también un carácter excepcional ${ }^{15}$. Creo, sin embargo, que existen otras razones, po-

13 "En la compañía hay soldado/que canta por excelencia;/y la Chispa, que es mi alcaida/del boliche, es la primera/mujer en jacarear"' (II, vv. 134-138).

14 Véase M. Frenk, Cotpus..., núm. 2281.

15 Aunque, como he observado, la Chispa no vuelve a dar ninguna muestra concreta de su talento de mujer única en el arte de jacarear, el tema se vuelve a tocar con mucha sutileza en un momento próximo ya al desenlace, cuando le promete a Rebolledo elevarle a la dignidad de personaje central de una jácara si sabe resistir el tormento sin cantar: "Rebolledo, determina/negarlo punto por punto;/serás, si niegas, asunto/para una jacarandina que cantaré"' (III, vv. 607-611). 
siblemente convergentes, para que el romance cantado a dos voces por Rebolledo y por la Chispa en la primera jornada no se sitúe en la línea de la jacarandina mencionada en los pareados que encabezan la canción. Y esto es lo que me incita a volver ahora, en cierto sentido, a mi punto de partida.

Lo haré apoyándome, en primer lugar, sobre lo que se nos alcanza de la historia o de la prehistoria respectiva de las varias piezas que entran en el mosaico formado por el dúo de Rebolledo y de la Chispa. Este dúo ofrece, eh efecto, la particularidad de estar en su totalidad recogido en el ya citado Corpus de la antigua lírica popular. "Particularidad" que comparte, por supuesto, con un número relativamente elevado de canciones que figuran en obras dramáticas del Siglo de Oro, pero que es, en cambio, otro rasgo distintivo que lo separa de las usuales celebraciones festivas de la vida hampesca. Ahora bien, si nos fijamos en la documentación que la eruditísima presentadora del Corpus ha podido reunir sobre las diferentes partes o secuencias distinguidas por ella en este dúo, observamos con facilidad que, mientras la tal documentación es poco abundante e mcluso escasea para los dos pareados del comienzo y para la primera cuarteta del romance ${ }^{16}$, es notablemente más copiosa para la segunda cuarteta, a saber, para la ya citada celebración entre onírica y festiva del pan abundante y de la superioridad de la carne fina de la gallina sobre la del carnero, más dura de digerir. Sabemos en efecto, gracias a Margit Frenk, que los versos 109-110 de la canción calderoniana ("Vaya y venga la tabla al horno,/ y a mí no me falte pan') gozaban ya previamente a 1639 de la suficiente popularidad para que se volvieran a lo divino, y que la totalidad de la copla aparece por otra parte parodiada en un entremés de Quiñones de Benavente, recogido en una recopilación que se publica en 1645. Pero el dato indudablemente más valioso del que disponemos a propósito de esta segunda cuarteta, dato que obliga nuevamente a distinguir entre los versos 109-110 y los siguientes, es que dos versiones más o menos parecidas a la calderoniana se encuentran ya repertoriadas en Correas ${ }^{17}$.

${ }^{16}$ Para los dos pareados, no pueden citarse más correspondencias que las dos que han sido examinadas previamente en el presente trabajo. En cuanto a la cuarteta en que se prodiga el vocabulario militar ("Vaya a la guerra el alférez/y embárquese el capitán..."), es preciso distinguir entre los dos primeros versos, que sólo se encuentran documentados en El alcalde, y los siguientes, que aparecen citados en una suelta sin fecha de Matos Fragoso.

${ }^{17}$ Las dos variantes que figuran en el Vocabulario de refranes son "Vaya y 
No es éste el único caso en el que se advierte que existe una relación entre el personaje de la Chispa y el material recopilado en el Vocabulario de refranes. Me parece en particular muy cargado de sentido que encontremos entre las primeras palabras que pronuncia la Chispa, junto a unos vulgarismos que en seguida permiten identificarla como una figura del mundo marginal, una alusión a un dicho famoso, recogido y glosado por el maestro salmantino. Esto es en efecto lo que sucede cuando, después de haberse dirigido a Rebolledo en una muy estilizada clave hampesca ("Seor Rebolledo, por mí/vuecé no se aflija, no", vv. 6566), afirma la Chispa que, según bien sabe su interlocutor, ella tiene desde que nació "barbada el alma". Esta pintoresca manera de contrastar cuerpo y alma es famosa, según he indicado, aunque no por que se encuentre repertoriada y glosada de una manera singularmente edificante por Correas ${ }^{18}$, sino por haber sido aprovechada por Cervantes en la Segunda parte del Quijote ${ }^{19}$.

venga la pala al horno, que nunca le falte pan' y "Vaya y venga la pala al horno, que no hay bocado" (514b). Sabido es que Combet da por terminada la redacción del borrador del Vocabulario de refranes en 1627 . Véase su edición, p. vi.

${ }^{18} \mathrm{El}$ dicho figura en CORREAS con las siguientes variantes: "En el alma tengamos barbas, y basta" (ed. cit., p. 122a); "La (el) alma tenga barbas"; "Y en el alma tengamos barbas" (ibid., p. 181a). El comentario figura entre los más extensos del Vocabulario de refranes y entra en violento contraste con la aplicación del dicho al caso específico de la Chispa. Luego de haber indicado que lo que se señala con estas palabras es que "no se ha de mirar tanto por el ornato exterior como por el interior de entendimiento y virtud', Correas las atribuye en efecto a un "gentilhombre mozo estudiante, natural de Ávila, llamado Ortiz, que teniendo llamamientos para religión, le hacía estorbo haber de cortar buena barba y bigotes que tenía'. Gran parte de la anécdota está dedicada a presentar muy en detalle la mortificación que supone el sacrificio de semejantes ornatos personales y el final da a entender que éste no fue sino el primer paso dado para la realización de un destino gloriosamente ejemplar: "Al cabo entró en la Compañía de Jesús, y siendo enviado al Japón con otros, fue martirizado en el mar por Holandeses, preso en el navío en que iban antes de desembarcar, y capitán de los otros en el martirio".

19 " [. . . ] barbada y con bigotes tenga yo mi alma cuando desta vida vaya, que es lo que importa". Estas palabras forman parte de una respuesta de Sancho a la Condesa Trifaldi ( $D Q$, II, 38; ed. de Luis A. Murillo, Clásicos Castalia, Madrid, t. 2, p. 331). Según apuntan varios anotadores del Quijote, el dicho se encuentra ya en SÁnchez de la BALLesta, quien lo glosa de la manera siguiente: "fue manera de decir de un cuerdo eunuco, significando que no se ha de procurar tanto el ornamento del cuerpo como el del alma' "Dictionario de vocablos castellanos aplicados a la propiedad latina, Salamanca, 1587, p. 370. En cuanto a Gaspar Lucas Hidalgo, citado por F. Rodríguez Marín, vemos 
Volviendo a la escena inicial de $E l$ alcalde ${ }^{20}$, creo que se ha de resaltar la perfecta distribución de los momentos en que se hace uso de estas referencias proverbiales: mientras que la primera declaración de la Chispa encierra en su comienzo, según acabamos de ver, una alusión a un dicho proverbial, su participación personal en el dúo que interpreta con Rebolledo se cierra, por otro lado, con la presentación del fragmento de más indudable raíz popular de todo el interludio. También es notable el relieve con el que se encarga, mucho más adelante, de poner punto final a la escena en la que los soldados se disponen a salir tempranamente de Zalamea, según se les ha ordenado tras la agitada noche de la serenata, remitiendo expresamente a otro cantar proverbializado, que ella presenta en estas circunstancias como propio de su repertorio personal: "Y yo veo agora/por qué en el mundo he cantado/ «que el amor del soldado/no dura una hora»" 21 . Para que quede completo estre breve repaso del uso calderoniano de un material proverbial para caracterizar a este singular personaje femenino, agregaré que, según recuerdan sin duda muchos lectores, a la Chispa le corresponde por fin aportar el contrapunto humorístico de un refrán en medio de la escena en que, con los preparativos del rapto, está ya muy próximo a quedar trágicamente sellado el destino de Isabel ${ }^{22}$.

Si se exceptúa este último chiste, que está en la línea de lo que podría decir cualquier gracioso, los demás usos del refrán que entran en la elaboración del personaje de la Chispa son profundamente originales. No me detendré aquí, por creer que merece un estudio aparte, en el caso específico del dicho tradicionalmente

que lo atribuye a un "mancebo encogido y mortificado en su condición", que, como el estudiante de Correas, habla "muy a lo devoto", pero que, a diferencia de él, es a los veintiocho años prácticamente imberbe y se convierte por lo mismo en blanco de muy mortificantes vayas (Diálogos de apacible entretenimiento, III, 3 ; BAE, t. 36 , p. $308 \mathrm{~b}$ ).

20 Tomo la libertad de hablar de escenas, pese a los reparos justificados que ha suscitado el empleo de esta terminología. Advierto que, según se echa de ver en seguida, el vocablo no tiene para mí ningún valor técnico.

${ }^{21}$ La versión recogida en CORREAS es en este caso más extensa: "El amor del soldado no es más de una ora, ke en tokando la kaxa: ¡i a Dios, señora!" (Vocabulario de refranes, ed. de Louis Combet, Bordeaux, 1967, p. 86a). Cionservo excepcionalmente la grafía original, por tener dudas acerca de la interpretación que se le ha de dar al final ("y jadiós!" o "id a Dios" = id con Dios).

22 "LA CHISPA [comentando palabras de Rebolledo, quien acaba de pedirle que guarde las capas de los que se preparan a reñir con Crespo y con su gente] Que es del reñir, imagino,/la gala el guardar la ropa,/aunque del nadar se dijo", vv. 823-825. 
asignado a un eunuco, a un hombre imberbe o a quien se corta barbas y bigotes por motivos religiosos, y que la Chispa se aplica a sí misma para dar idea de su "hombría". Insistiré, en cambio, sobre el carácter excepcional de sus intervenciones lírico-proverbiales, que hacen de ella un personaje aparte ${ }^{23}$. No basta, sin embargo, observar esta singularidad de la Chispa, como cantadora más que como decidora de refranes. Ha llegado el momento de enlazar por fin estas laberínticas consideraciones sobre el refrán con lo que al principio se dijo cuando, en la línea de las observaciones de José Luis Alonso, se resaltó que la jácara cantada al comienzo de El alcalde de Zalamea no encaja de ninguna manera en la tradición de la jácara-germana. Si, como entonces también se observó, el vacío que deja la ausencia de la materia hampesca tiende a llenarse en los dos pareados con las pintorescas sonoridades de unas onomatopeyas de sabor tradicional, la solución ideada para llenar el mismo vacío en el romance es otra. Lo que se canta a partir del verso 105 ("Vaya a la guerra el alférez [. . . ]") es una jácara donde lo hampesco ha sido sustituido por lo proverbial. Es cierto que en ningún refranero se hallarán coincidencias concretas con los versos de la primera cuarteta, en la que se prodigan, según hemos visto, las marcas léxicas propias de la vida militar. Pero la temática que éstos desarrollan se encuentra plenamente en la línea de los contrastes que, desde que comienzan a reclutarse masivamente villanos que asientan a soldada, oponen bajo la forma de dichos proverbializados la actitud de los mandos a los de la tropa ("El capitán, galgo; y los soldados, liebres"; "El capitán, león;

${ }^{23}$ Estoy de acuerdo con Jean Canavaggio en pensar que el trabajo pionero de MARGIT FRENK sobre los "Refranes cantados y cantares proverbializados", recogido en sus Estudios sobre lírica antigua, Castalia, Madrid, 1978, pp. 154-171 está pidiendo prolongaciones, y que una de las posibles prolongaciones sería un estudio del tratamiento que en Calderón y en otros autores reciben los refranes de clara filiación con la lírica tradicional y los que en cambio son, o parecen ser, más propiamente "dichos". Véase la conclusión de CA. NAVAGGIO a su finísimo trabajo titulado 'Calderón entre refranero y comedia: de refrán a enredo", en Actas del Congreso Internacional sobre Calderón y el teatro español del Siglo de Oro, C.S.I.C., Madrid, 1983, t. 1, pp. 393-402. Estas consideraciones no se recogen en la versión reelaborada que con idéntico título se publicó paralelamente en Aureum Saeculum Hispanum, Festschrift für Hans Flashe, Franz Steiner Verlag, Wiesbaden, 1983. MARC VITSE habla por su parte en términos más generales del problema de las relaciones entre Calderón y el refranero y del capítulo que, sobre este tema, "queda aún por escribir". Véanse sus útiles apostillas y sus comentarios a la edición crítica de Cada uno para sí, realizada por José María Ruano la Haza, en Criticón, 1984, núm. 27, pp. 109-128. 
y los soldados, ciervos", ambos en Correas, p. 100a). Y la rela ción misma que Calderón establece festivamente entre el desec de que otros maten a los enemigos en el campo de batalla, o sea entre el deseo personal de ser gallina, y el de ver sacrificadas er beneficio propio a las gallinas del corral, puede verse como uná ilustración directa de No matará cosa que no sea de comer, dicho cor el cual, según aclara Correas, era corriente tacharle a uno de sol. dado cobarde (p. 261a).

Se me podrá objetar que estos temas que así encuentran ex. presión en forma proverbial son tópicos muy gastados, que figu. ran en las denuncias o en las sátiras que suele suscitar la vida mi. litar. Pero no todos los tópicos se llegan a proverbializar. Por curioso que pueda parecer, no dejan por ejemplo huella en e refranero ni la bien conocida imagen del soldado que jura y pro. fiere blasfemias, ni la no menos familiar del soldado jugador ${ }^{24}$ Lo que se observa en la extensa presentación de la soldadesca cor la que se inicia El alcalde de Zalamea es que Calderón reserva all a estas dos categorías de imágenes ampliamente divulgadas, la: proverbializadas y las no proverbializadas, una suerte distinta. És. tas se dramatizan y se integran, por decirlo así, en las vivencias personales de Rebolledo, según podemos ver por el magnífico ejem. plo de la blasfemia que suelta en el momento mismo en que st inicia la obra ${ }^{25}$. También puede servir para ilustrar mi tesis e. destacado lugar que el divulgadísimo tema de los ataques contra la corrupción interna de la administración militar llega a tenes en sus manifestaciones de descontento (vv. 17-36). Éste es en efeetc otro caso de tema que se convierte en lugar común, al menos a partir de las guerras de Italia, pero sin que este uso reiterado desemboque en su proverbialización. El dramaturgo se apoya en cambio directa o indirectamente en el refranero para el nivel in comparablemente más cargado de fantasía en el que Rebolledc no lleva ya, literalmente hablando, la voz cantante, puesto que a quien le corresponde tomar la iniciativa para cuanto allí se canta

${ }^{24}$ Para Francia, sé al menos de un refrán documentado en el siglo xvi en el que asoma el segundo de estos dos temas ("Cartes et dés, table de capi taine", citado por LE RoUX DE LINCY, Le livre des proverbes français, Paris, 1842 t. 2, p. 58). Quien desee tener una visión de conjunto de los refranes que, er el Vocabulario de refranes, se dedican a la vida militar y a los soldados podrá con sultar el índice que figura al final del libro de Lous COMBET, Recherches sui le "refranero" castillan, Les Belles Lettres, Paris, 1971, pp. 361-362.

25 " ¡Cuerpo de Cristo con quien/desta suerte hace marchar/de un lugai a otro lugar/sin dar refresco!" (vv. 1-4). 
es a la Chispa. Lo que se consigue mediante este juego y este cambio de nivel son efectos hasta cierto punto convergentes. Exactamente como se va aligerando el clima de tensión del principio cuando la Chispa saca sus castañetas y se echa a cantar, la dureza de trazo que podría quedar definitivamente asociada con esa mujer de costumbres poco edificantes se suaviza de un modo notable al hacer que, en lugar de cantar una jácara fiel a los cánones de la jácara-germana, salga cuando canta por vez primera por el camino de lo que ella llama festivamente la jacarandina ${ }^{26}$, y nos presente en realidad variaciones sobre refranes cantados o cantares proverbializados.

La conclusión que voy a dar al presente trabajo tendrá, según en seguida advertirá el lector, un tono muy personal. Esto se debe a las raíces muy íntimas de las preocupaciones de las que ha surgido este conjunto de observaciones. Si hay una línea homogénea en los trabajos que vengo publicando, creo que ésta radica en mi deseo de rescatar lo que la rutina incita a desechar y, paralelamente, aunando mis esfuerzos con los de otros investigadores, en el de dar dignidad a lo usualmente calificado de secundario, chico o menor. Es cierto que, en la actitud frente a lo popular y a lo marginado, ha habido grandes cambios, desde que estos temas, y los conceptos correspondientes, se pusieron de moda a finales de los años sesenta. Pero lo que se observa es que la tendencia a segregar en la práctica lo alto de lo bajo sigue dominando en la gran mayoría de la producción académica, pese a una recuperación de Bajtín que en muchos casos me parece de una profundidad cuestionable. Esto se refleja de una manera muy concreta en la suerte que le ha tocado a la pobre Chispilla la Bolichera y, más allá de su persona, al hilo conductor que en El alcalde de Zalamea representa la jácara -o, si se prefiere, y para seguirle mejor el humor a Calderón-, al hilo conductor de la jacarandina. En años en que sabemos que el público de los corrales está tan encaprichado con las jácaras que las está reclamando a voces ${ }^{27}$, llama la atención la brillantez y la finura con que Calderón — que por otra parte está jugando con la poética del género en un entremés suyo

${ }^{26}$ Más que en la flor de la jacarandina, estoy pensando, al decir esto, en un comentario esclarecedor de la Chispa sobre su propia canción: "Hoy saber intento/por qué dijo, ¡voto a tal!,/aquella jacarandina:/"Huéspeda, máteme una gallina, /que el cordero me hace mals"' (vv. 155-160).

${ }^{27}$ Véanse los testimonios aducidos por Jean Sentaurens en "Bailes y entremeses en los escenarios sevillanos de los siglos XVI y XVII [...]", en El teatro menor en España a partir del siglo xvi, C.S.I.C., Madrid, 1983, p. 83. 
y en el baile que lo prolonga- convierte otras variaciones suyas sobre el mismo tema en uno de los ejes constructivos de su gran drama "rural". Eje del que conviene además tener presente que es en este caso musical. Volvemos, a este propósito, a tropezar con el problema de la segregación entre lo alto y lo bajo que ha quedado mencionado en las líneas que preceden. A nivel teórico, es un hecho bien conocido que la música ocupa un lugar de primer plano en la dramaturgia calderoniana. El libro de Edward M. Wilson y Jack Sage está abundantemente citado, y en particular en las ediciones que ponen las obras más famosas del autor al alcance del público no especializado ${ }^{28}$. Cuando se celebra el tercer centenario de la muerte del escritor, una prestigiosa institución barcelonesa saca una tirada aparte de Miquel Querol sobre La música en el teatro de Calderón y la distribuye muy generosamente entre los hispanistas ${ }^{29}$. El tema se estudia, por fin, en relación con las obras escritas expresamente para ser cantadas, a modo de libretos. Pero, al tratarse de un género menor, el significado de la ininterrumpida presencia de la línea melódica de la jácara en $E l$ alcalde $e^{30} \mathrm{ni}$ siquiera se menciona en los intentos hechos para dar una visión cabal de su estructura ${ }^{31}$. Y esto, pese a ser este nivel concreto donde mejor se puede apreciar la capacidad de invención de un artista.

Más personales aún son las circunstancias que voy ahora a mencionar. Mi primer contacto con El alcalde de Zalamea, no de lectora, sino de espectadora, tuvo lugar en 1961, año en el que vi la obra en una escenificación de Jean Vilar. Todo, al parecer,

${ }^{28}$ Edward M. Wilson y Jack Sage, Poesías líricas en las obras dramáticas de Calderón, Tamesis, London, 1964.

${ }^{29}$ Diputació de Barcelona, Institut del Teatre, 1981.

${ }^{30}$ Presencia ilustrada, en la tercera jornada, por la ya mencionada promesa hecha a Rebolledo de convertir su entereza en "asunto para una jacarandina" (véase la n. 15) y por la breve pero original variación sobre las posibilidades polisémicas del verbo cantar (vv. 627-634). El hecho de que las voces de Rebolledo y de la Chispa sean las últimas en resonar antes que Crespo se dirija clásicamente al público para pedirle que perdone los defectos de la obra encaja, por supuesto, en la tradición de las intervenciones finales del gracioso. Lo que le da interés es la forma en que enlaza con los resurgimientos del tema musical a lo largo de las tres jornadas.

${ }^{31}$ Como podrá comprobar quien vuelva a leer el clásico ensayo de ALExander A. PARKer, "La estructura dramática de El alcalde de Zalamea", en Homenaje a Casalduero, Credos, Madrid, 1972, pp. 411-417, o a consultar la no menos clásica guía de Premraj R. K. Halkoree, Grant \& Cutler, London, 1972. En la exposición del tema que presenta en su introducción, Díez Borque hace hincapié en su deuda con estos dos autores. 
se reunía para impedirme apreciar la presencia de los problemas a los que aquí me he referido. Además de representarse la obra en francés, las lagunas de mi formación de hispanista eran por esas fechas auténticamente abismales. Algo sabía, o comenzaba a saber, de refranes; estaba fresca en mí la fuerte impresión, nunca borrada, desde luego, que me habían producido las muestras de poesía popular recogidas por Dámaso Alonso y José Manuel Blecua en su antología; de las jácaras, en cambio, nunca había oído hablar. $Y$, sin embargo, tuve en seguida la sensación de que el comienzo del drama, con el dúo de la Chispa y Rebolledo, encerraba algo que estaba pidiendo explicación, un enigma sobre el que algún día tendría que meditar. El fruto de esta meditación que quedó tanto tiempo aplazada, se lo ofrezco ahora a quien, a finales de los mismos años sesenta, aceptó con gran generosidad una primera colaboración mía para la prestigiosa revista de la que era entonces director.

Monique Joly Université de Lille III 
